\title{
Melt Stirring of Nodular Cast Iron
}

\author{
Fareed Ashraf Khan ${ }^{1, a^{*}}$, Hasse Fredriksson ${ }^{1, b}$ \\ KTH Royal Institute of Technology, Department of Materials Science and Engineering, Casting \\ Division Brinellvägen 23, Stockholm, SE-100 44, Sweden \\ afareedak@kth.se, bhassef@kth.se
}

\begin{abstract}
Keywords: Melt stirring, Melt shearing, Turbulent flow of melt, Nodule count, Nodule distribution, Nodular Cast Iron, Ductile cast iron, Oxidation of melt, Nucleation in cast iron. Formation of Graphite strings.
\end{abstract}

\begin{abstract}
In this work the effects of stirring/vigorous shearing on matrix and graphite phase in nodular cast iron melt during solidification were studied. Several experiments were conducted for different cooling and stirring times. Samples were prepared and examinations of the microstructure were conducted and compared using Leica Optical Microscope and Scanning Electron Microscope (SEM). In addition, a chemical analysis of the graphite precipitate was performed using EDX equipment mounted on SEM. Oxidation of the melt and formation of oxide nuclei during stirring were observed and analysed. The influence of melt shearing on the structure, nodule count, distribution, area fraction and on overall graphite precipitation was observed and examined with the help of respective computer programs and softwares. Stirring resulted in increasing the nucleation sites for graphite precipitation thereby increasing the nodule count and area fraction of carbide and transforming the structure from pearlitic matrix to ferritic matrix.
\end{abstract}

\section{Introduction}

On a macro level, the casting process is a combination of fluid flow and heat transfer. Beside the mass transfer, the convection has an important role in heat transfer and causes rapid removal of superheat from the melt. Numerous flow mechanisms occur during pouring of melt into the cast. Im et al. [1] have mentioned the concurrent filling in a two-dimensional square cavity. Various flow systems involved during such processes are: (i) filling via gating system. (ii) Momentum of flow due to incoming melt and (iii) natural convection due to thermal and density gradient [2].

In continuous casting and during semisolid processing in thixotropic process [3], the convection significantly affects the solid-liquid interface formation. Swaminathan et al. [4] have tracked the air/liquid interface during pouring. The convection in the melt stream during teeming in mold causes turbulence, in which air bubbles can be trapped and can enhance the oxidation of the melt and transport these oxides further into the mold cavity.

Inoculation can be described as the addition of nuclei to the molten cast iron, thereby increasing the number of nucleation sites on which eutectic graphite precipitates during the solidification process. The main aim is to influence the microstructure in a specific way. Nuclei are usually fine oxide particles, $\leq 4 \mu \mathrm{m}$ in size, which act as graphite crystallization sites. There are several theories concerning inoculation, out of which the oxide nucleation is thought to be the most appropriate one, $[9,10,11]$. As per this theory, $\mathrm{SiO}_{2}$ particles are precipitated, and act as nuclei. Precipitation of these particles is dependent on the formation of other foreign nuclei. These foreign nuclei are created by the elements which have high affinity for oxygen. $\mathrm{Mg}$ is added to the cast iron as an inoculant [5]. $\mathrm{Mg}$ has high affinity for oxygen and will readily react with the absorbed oxygen in the melt and form $\mathrm{MgO}$ particles. These particles act as nuclei on which graphite deposits. The ductility, strength and toughness of nodular cast iron is attributed to the size and spacial distribution of graphite phase [5].

In a previous study [14] the cast structure had been analysed in downhill and uphill casted insert. Samples from different positions in the cross sections of the inserts were investigated. It was observed that the average number of nodules per $\mathrm{mm}^{2}$ in downhill casting is $38 \%$ more than in uphill casting. It was proposed that since the turbulence in downhill casting as compared to the 
uphill casting is very high, so beside the fact that an inert gas atmosphere is created while casting, the magnesium in the melt has a very high affinity for oxygen and enough oxygen is still present in the mould so more oxides are formed due to the violent movement of melt, which act as nuclei for graphite nodule precipitation. We also observed string-like oxide structures on which graphite had precipitated.

Convection in the melt during solidification can change the aspect of heat and mass transfer and also increases the interaction of oxygen with the melt, which can affects the microstructure and distribution of the graphite. [8]. for this purpose we have mechanically stirred the melt during solidification for different durations and investigated the formation of different oxides and its effect on the graphite morphology.

\section{Methodology and Experimental Work}

\section{Equipment}

Fig.1 illustrates the experimental arrangement. Stirring was conducted by a quartzes rod fitted on a variable speed drilling machine. Quartz cruicibles $90 \mathrm{~mm}$ length and $28 \mathrm{~mm}$ internal dia were placed in graphite susceptor. Special resistance furnaces were designed and developed to control the melt temperature and the cooling rate during stirring. Two different furnaces were built, the furnace initially built had a limitation of heating the crucibles to $650^{\circ} \mathrm{C}$ and could hold only one cruicible at a time. Some experiments were conducted using a resistance furnace which could hold two crucibles at a time, stirring was conducted in one of the crucibles and other was used as a reference to compare the un-stirred structure. The crucibles could be heated to $1200^{\circ} \mathrm{C}$.

Experiments were conducted at Roslagsgjuteriet AB in Herräng, Sweden. Table 1 gives the composition of the melt. The melt temperature during pouring in the mold was approximately $1200^{\circ} \mathrm{C}$. The samples were stirred at $2100 \mathrm{RPM}$.

Table. 1 Composition of the melt.

\begin{tabular}{|l|l|l|l|l|l|l|l|l|l|l|l|l|}
\hline $\mathrm{C}$ & $\mathrm{Si}$ & $\mathrm{Mn}$ & $\mathrm{P}$ & $\mathrm{S}$ & $\mathrm{Cu}$ & $\mathrm{Cr}$ & $\mathrm{Mg}$ & $\mathrm{Mo}$ & $\mathrm{Ni}$ & $\mathrm{Al}$ & $\mathrm{V}$ & $\mathrm{T}$ \\
\hline 3.71 & 1.95 & 0.17 & 0.01 & 0.01 & 0.02 & 0.03 & 0.05 & 0.00 & 0.05 & 0.01 & 0.01 & 0.01 \\
\hline
\end{tabular}

Details of the experimental conditions are given in Table 2. The crucibles were preheated at different temperatures to vary the solidification time and thereby altering the stirring time. All the samples were initially cooled in the furnace till around $600{ }^{\circ} \mathrm{C}$ and then the crucibles were taken out of the furnace and cooled in ambient conditions. Graphite was added to the ROS 8 to see the carbon content and study its effect.

Table. 2 Experimental condition.

\begin{tabular}{|l|l|l|l|l|l|}
\hline Sample \# & $\begin{array}{l}\text { Stirring } \\
\text { Time }\end{array}$ & Inoculant & $\begin{array}{l}\text { Graphite } \\
\text { Added }\end{array}$ & $\begin{array}{l}\text { Crucible } \\
\text { temp. before } \\
\text { pouring }\end{array}$ & Comments \\
\hline ROS 1 & no stirring & added & - & - & As cast \\
\hline ROS 2 & $10 \mathrm{sec}$ & added & - & Room temp. & - \\
\hline ROS 3 & $27 \mathrm{sec}$ & added & - & Room temp. & - \\
\hline ROS 4 & $28 \mathrm{sec}$ & added & - & $450^{\circ} \mathrm{C}$ & - \\
\hline ROS 5 & $42 \mathrm{sec}$. & added & - & $650^{\circ} \mathrm{C}$ & - \\
\hline ROS 6 & 85 seconds & added & - & $800^{\circ} \mathrm{C}$ & - \\
\hline ROS 8 & 100 seconds & added & 15 grams & $1000^{\circ} \mathrm{C}$ & - \\
\hline
\end{tabular}

\section{Investigation}

Three cross sections from top, middle and bottom areas were cut, mounted and prepared for optical microscopy by successive grinding and then polished by 3 and $1 \mu \mathrm{m}$ diamond paste and 
etched with $2 \%$ Nital. Microprobe analysis was conducted using Hitachi SEM and ESPIRIT 2.0 software was used to analyze each peak that appeared during micro analysis.

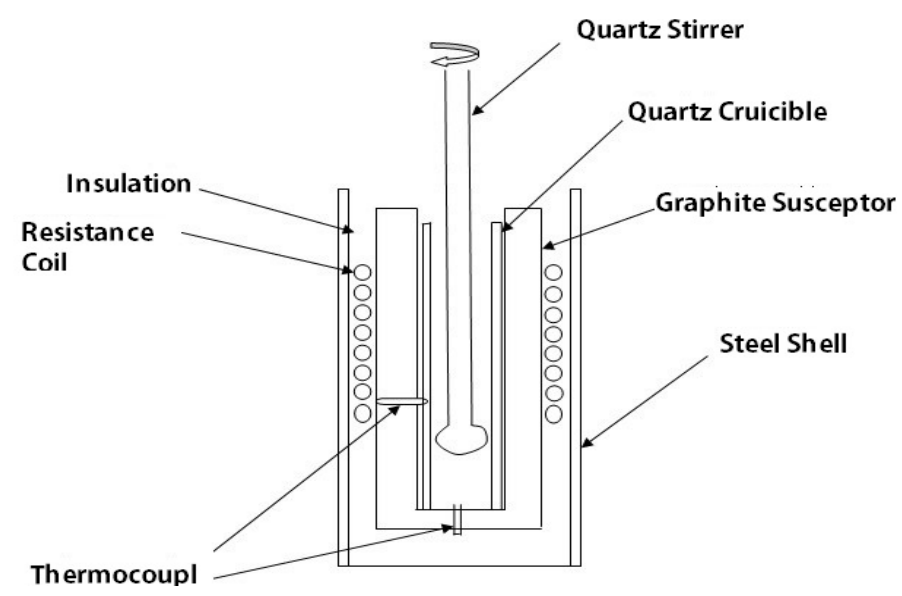

Fig. 1 Schematic illustration of the configuration of the equipment during experiments.

The representative area was carefully selected for observation; in stirred samples, the areas most affected by stirring, i.e. the lower central areas were selected. Micrographs of un-etched samples were taken using Scattered Electron Microscope (SEM).

The size distribution, count and \% area fraction were measured using Leica Qwin standard version 3.51. The count and area fraction of all graphite particle shapes and nodular shapes were recorded separately as per ISO standards. The nodule sizes which were recorded in the given samples were quite small and were distributed in a comparatively narrow size range, but the size ranges used by ASTM for classification of nodule are very wide and are not that descriptive; for this reason, we used narrow size ranges which were more expressive.

\section{Results}

Figs. $2(a, b)$ show the matrix of an unstirred sample, the inoculant was added prior to pouring in the mould. Graphite nodules are surrounded by ferrite in a pearlitic matrix. Carbides are present in fishbone and other typical morphologies.

Sample ROS 2 number of nodules has increased but several of these nodules have morphologies other than nodular Fig.3a. Most of the nodules are surrounded by ferrite grains but some last precipitated nodules are also surrounded by pearlite. The matrix is pearlitic. Some stringy elongated structures are present in Fig. 3b. Fine flakes of graphite are also observed. Some agglomeration of graphite nodules was also observed. Very few carbides are present in the area most affected by stirring. Mostly carbides including ledeburite are present along the wall of the crucible, the area which is less affected by stirring. Sample ROS 3 as shown in Fig. 4 (a, b), mostly the structure is similar to the sample Ros 2. But we see the increase in carbides precipitated in the most affected area and also an increase in the amount of chunky graphite. Sample ROS 4 shown in Fig.5 (a, b). The structure has completely transformed from a pearlitic matrix to a ferritic matrix with a few smaller intergranular pearlitic precipitations.

Precipitation of graphite nodules has increased in the stirred area and we see large number of small nodules formed along with other morphologies (Fig. 5a). The number of nodules has increased and we see quite a few large and small agglomerated nodules. Large string-like structures, both branched and straight can also be seen. Very few carbides are present in the affected area, but we can observe the precipitation of carbides along the wall of the crucible; some precipitation of graphite was observed within these carbides. Sample ROS 5 as shown in Fig. $6(\mathrm{a}, \mathrm{b})$ the matrix is completely ferritic. No pearlitic precipitation was observed. Ferrite matrix has very fine grains. Very few carbides has precipitated as compared to sample ROS 4. Precipitation of some undercooled/vermicular graphite was observed (Fig. 6a). Fig.7 of sample ROS 6 shows a ferritic matrix. Most of the graphite has precipitated as flakes with some nodular and chunky precipitation. 

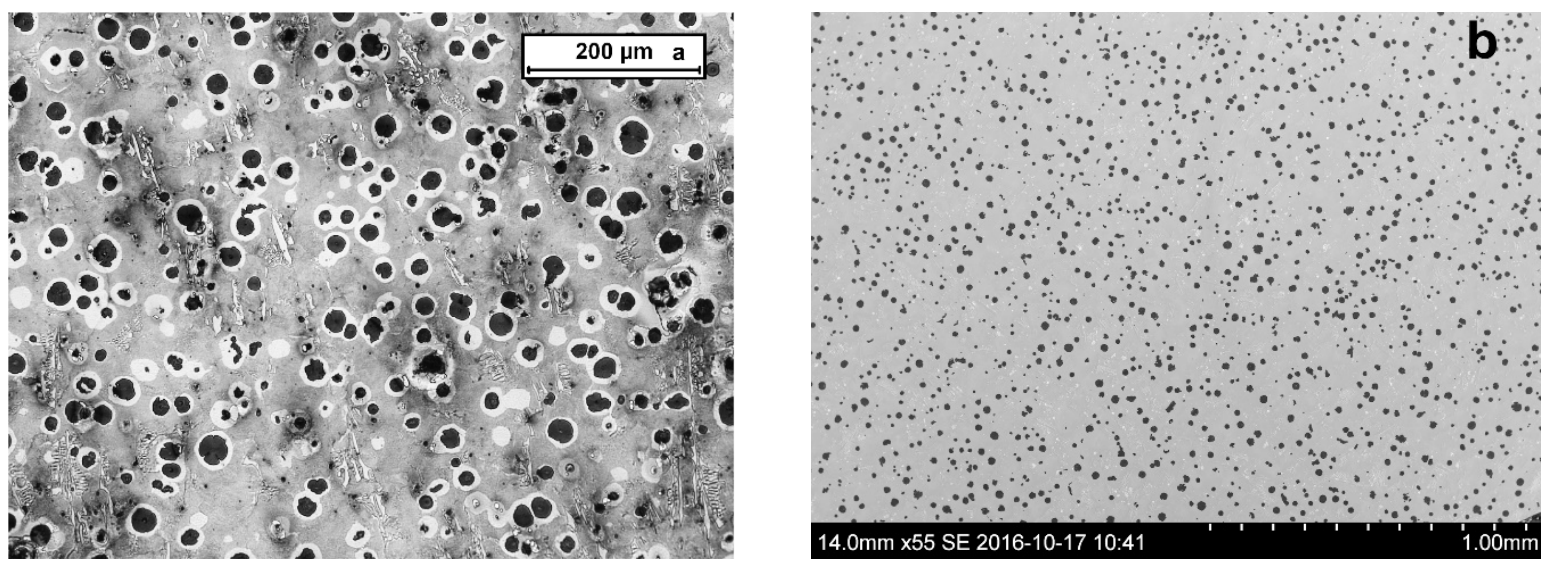

Fig. 2 (a,b), Sample ROS 1 a) LOM micrograph - Graphite nodules surrounded by ferrite in pearlitic matrix. Carbides have precipitated. b) SEM micrograph - Nodules in variable sizes are seen.
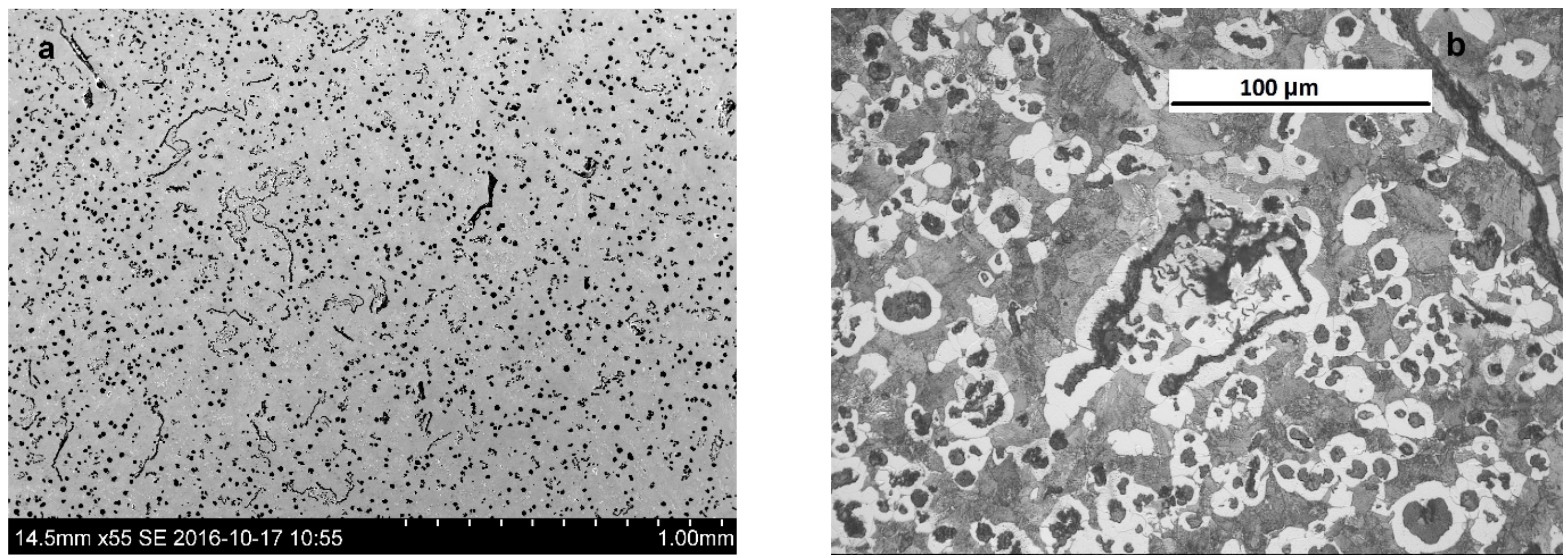

Fig. 3 (a,b) Sample ROS 2. a) SEM micrograph and b) LOM micrograph- Graphite nodules and stringy graphite surrounded by ferrite in pearlitic matrix. Some graphite flakes precipitated within ferrite phase.
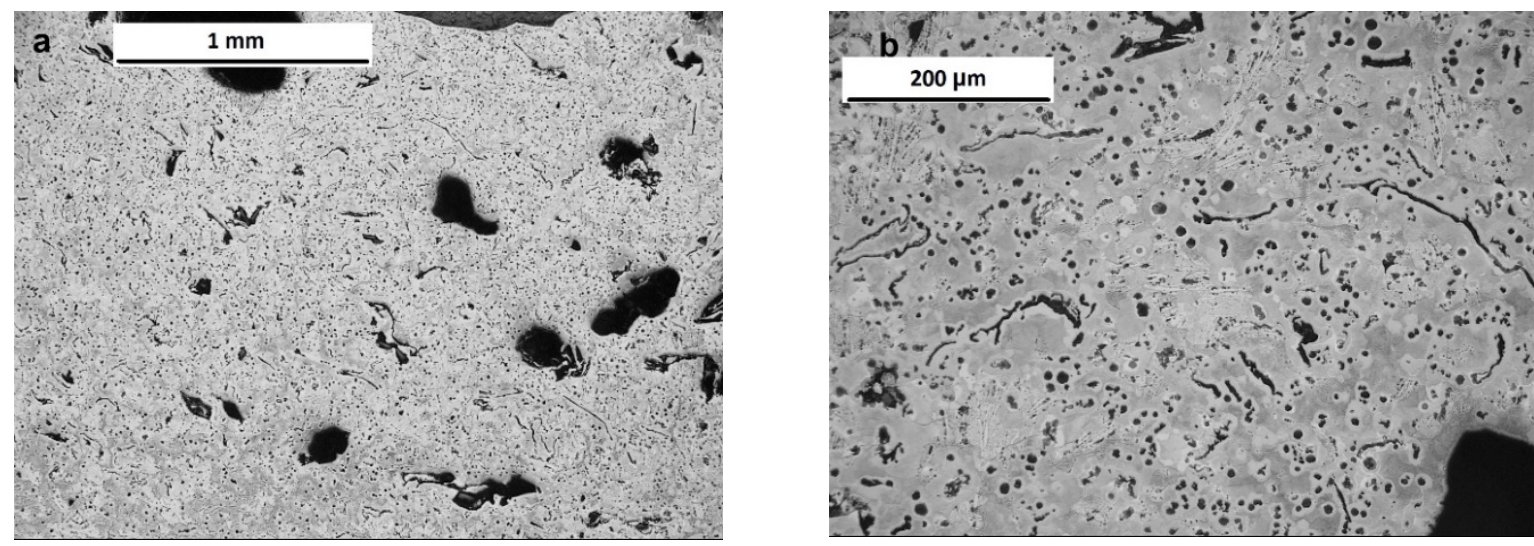

Fig. 4 (a,b) LOM micrographs, Sample ROS 3 Increase in nodule count. Compacted and stringy graphite have precipitated. Carbides fraction has increased. Pearlitic matrix.

The reference, which was cooled in the same conditions but without any stirring, shows precipitation of nodular graphite, some of them are significantly large. In Fig. 8 of sample ROS 8 shows that the matrix is ferritic, with large portion of graphite precipitated as under cooled graphite and some vermicular flakes, very few nodules are visible. 

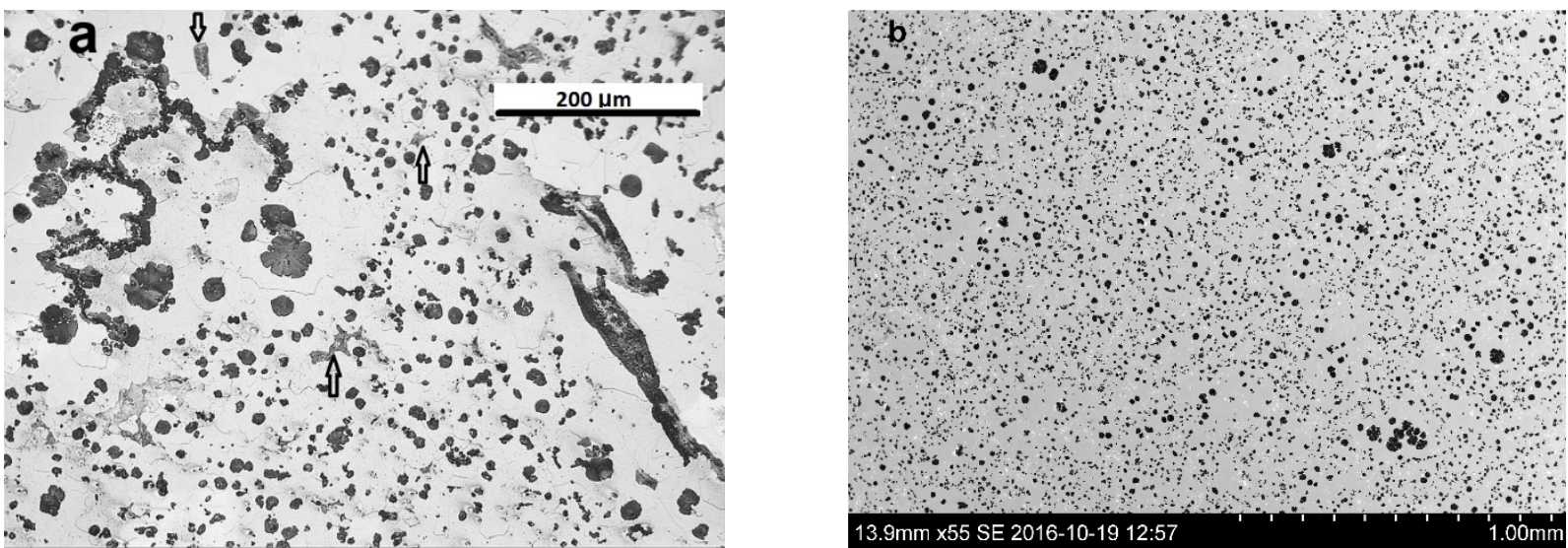

Fig. 5 (a,b) a) LOM micrograph, b) SEM micrograph, Sample ROS 4.Ferrite matrix. Small interdendritic pearlite precipitated which is marked by arrows.

Increased nodule count in variable sizes.
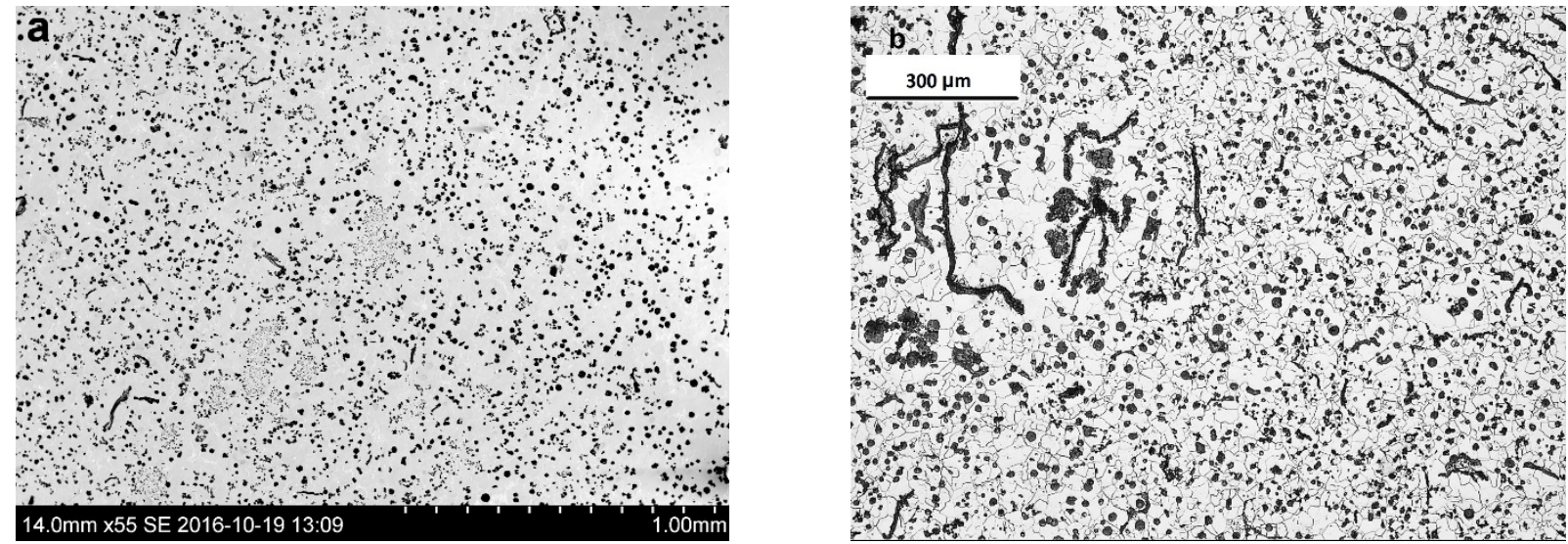

Fig. $6(a, b)$ a) SEM micrograph and b) LOM micrograph, Sample ROS 5. Fine grain ferritic matrix, no pearlitic precipitation observed. Fewer carbides have precipitated as compared to sample ROS 4.

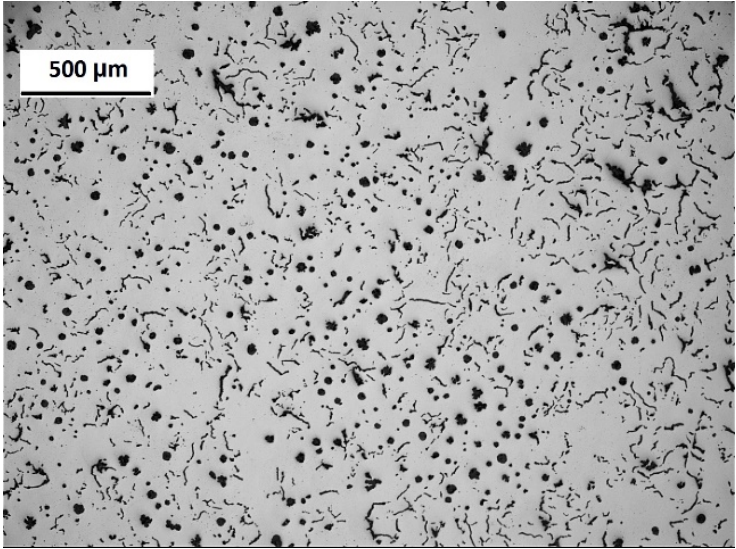

Fig. 7 LOM micrograph, Sample ROS 6.

Ferrite matrix, most of the graphite has precipitated as flake and compacted graphite

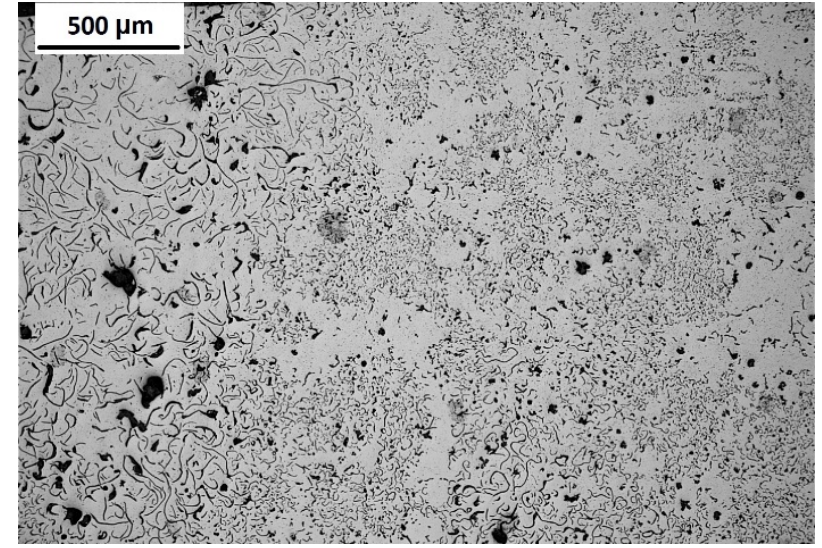

Fig. 8 LOM micrograph, Sample ROS 8. Ferrite matrix, graphite precipitated as under cooled graphite and some vermicular flakes

\section{Chemical analysis}

The micro analyses were conducted on the polished and etched sample using EDX. The line analysis conducted on the string like structure precipitated during solidification of the stirred melt. It was observed that the bulk of it consists of carbon whereas the central area of these cross-sections of strings contained either a mixture of $\mathrm{MgO}, \mathrm{FeO}$ and $\mathrm{SiO}_{2}$, or a complex oxide of $\mathrm{Mg} \mathrm{Fe}$ and $\mathrm{Si}$. Two types of strings were observed: rough-edged branched strings Fig. 9a and smooth-edged 
strings Fig. 10a. Figs. $9 \mathrm{~b}$ and $10 \mathrm{~b}$ shows two of the representative results of the line analysis of these string-like structures.
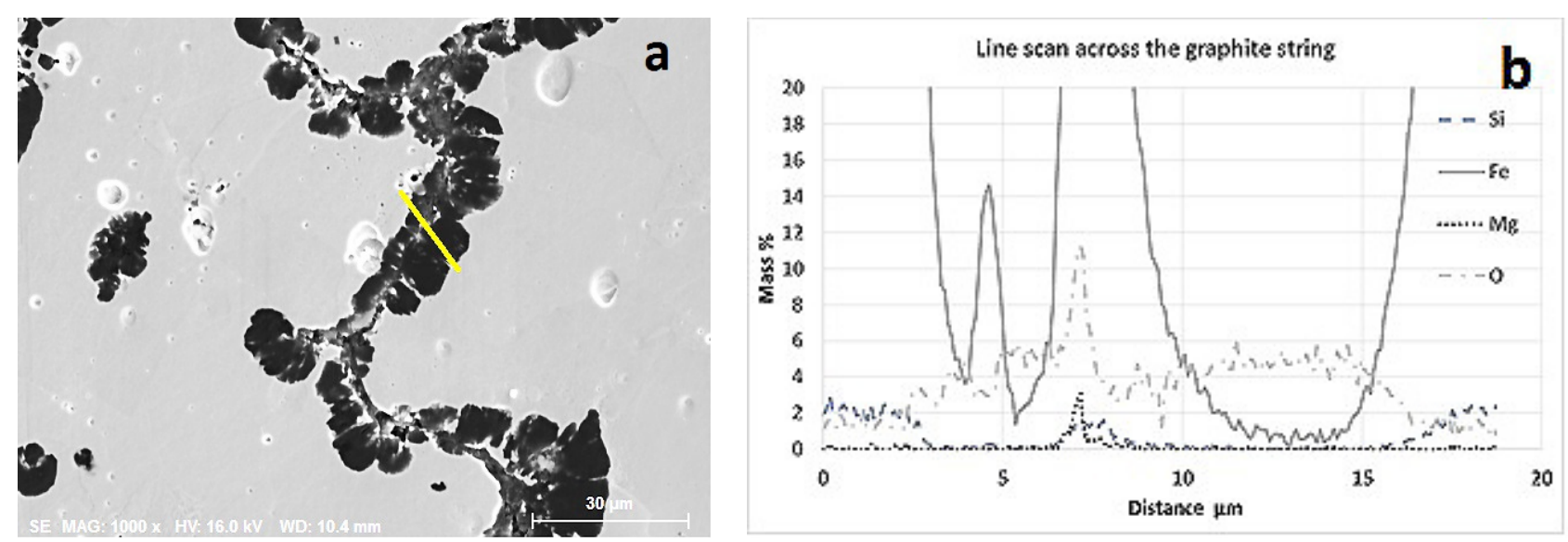

Fig. $9(a, b)$ a) Shows the line scan path, b) Result of line scan analysis of a branched string.
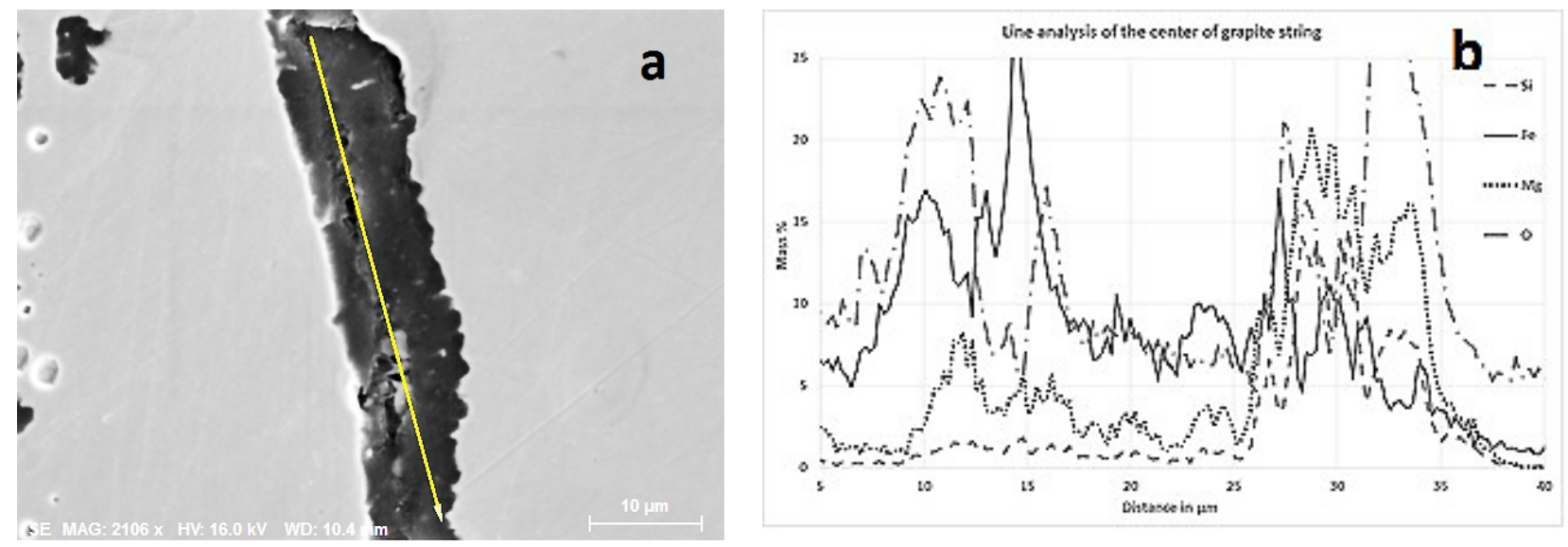

Fig. $10(a, b)$ a) Shows the line scan path, b) Result of line scan analysis of smooth surface string.

\section{Graphite morphology and distribution}

From the graphs in Fig 11 and 12, we can observe that the graphite nodule/particle count and area fraction has increased by increasing in stirring time and prior heating of the crucible. Since sample ROS 6 and ROS 8 graphite precipitated as flakes so they are not included in this analysis.

\section{Discussions}

We have observed that the count of nodules plus other particles has increased significantly in the stirred samples as compared to the non-stirred sample. This increase in the graphite particles / nodules precipitation can be caused due to the formation of oxides during stirring of the melt, due to increase interaction of melt with the environment which increases the oxygen content in the melt hence causing the precipitation of oxides. These oxide particles act as the nuclei for the growth of the graphite nodules. The precipitation of higher number of oxide particles increases the number of graphite precipitation sites. This causes increase in the nodule count. But the stirring also causes the evaporation of $\mathrm{Mg}$, hence decreasing the $\mathrm{Mg}$ content of the melt which causes precipitation of significant number of non-nodular graphite particulate [13]. 

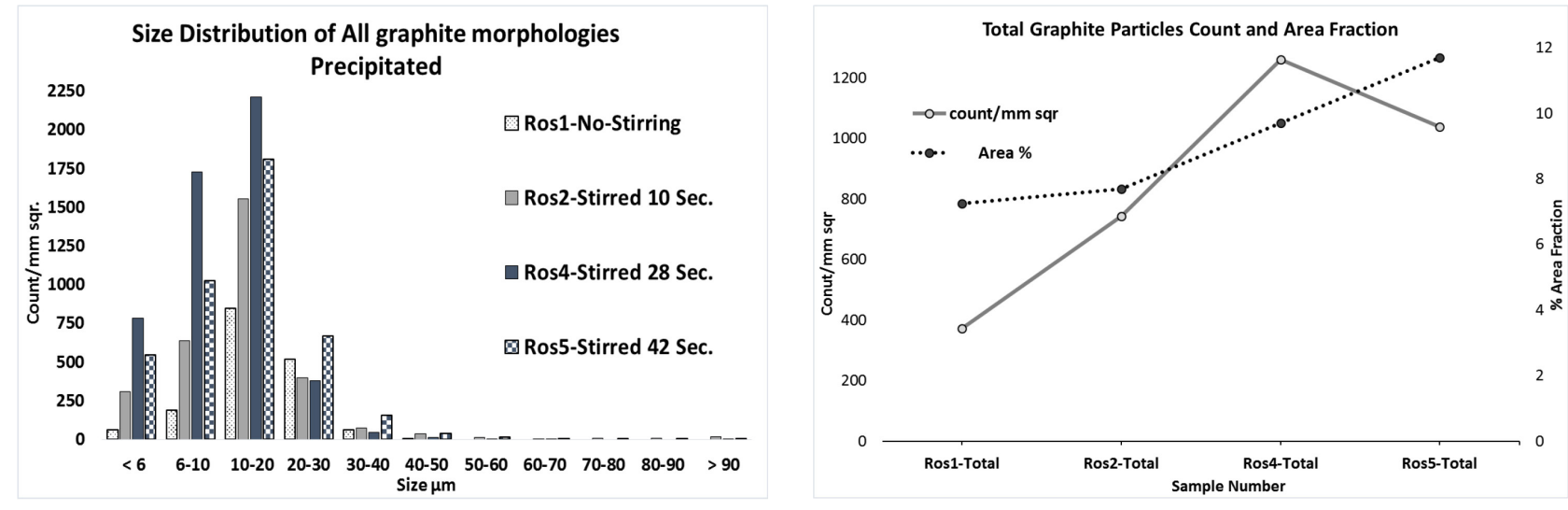

Fig. 11 Size distribution, count $/ \mathrm{mm} 2$ and the $\%$ area fraction of the all the graphite morphology precipitated.
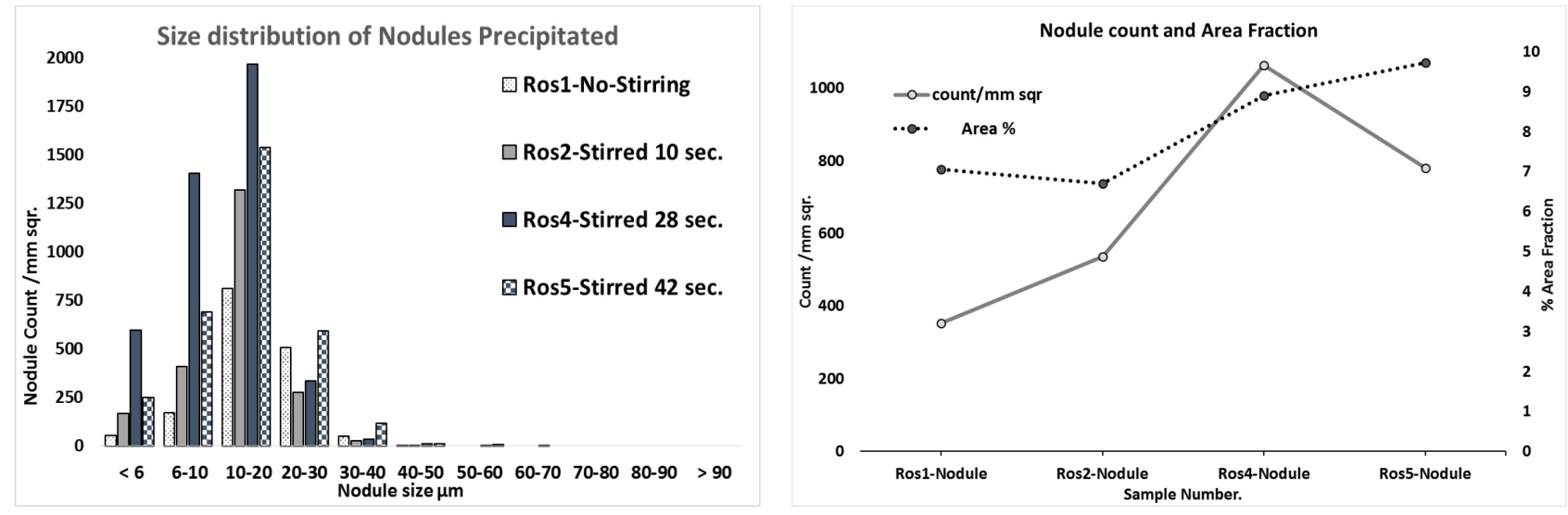

Fig. 12 Size distribution, count $/ \mathrm{mm}^{2}$ and the $\%$ area fraction of the graphite nodule precipitated.

String like structure had formed in the stirred sample. Which on chemical analysis showed that the oxides of $\mathrm{Mg}, \mathrm{Si}$ and $\mathrm{Fe}$, were present at the center of these strings and these oxides were surrounded by graphite. It seems that initially Fe and Si oxides precipitated in the form of strings at the surface, since at the surface non-equilibrium condition is created due to the interaction of the melt with the atmosphere. The $\mathrm{Fe}$ and $\mathrm{Si}$ oxides formed during this process are unstable oxides which decomposed and formed $\mathrm{MgO}$ [15]. During stirring these stringy oxides were dispersed in the bulk and some of them fragmented into smaller particles, these oxide particles and stringy oxides acted as nuclei for the deposition of graphite. The fact that there are no other graphite precipitated in the close vicinity of these strings shows that they were precipitated during early stages of solidification.

It will also be possible that small $\mathrm{MgO}$ particles are formed around the strings when they are transformed to more stable oxide. A process similar to the process illustrated by Fredriksson et al [12] when dissolving graphite in Fe-Ti alloy, giving a large number TiC nuclei. The oxide particles formed due to the stirring in the melt act as the inoculant and precipitate most of the graphite from the matrix discouraging the formation of cementite. Probably this is the reason why we see an increase in precipitation of graphite fraction by increasing the stirring time and decreasing the cooling rate. By increasing the precipitation of graphite particles we decrease the diffusion distance for carbon atoms, thereby increasing the graphite phase.

Transformation to ferritic matrix might be caused due to slow cooling of the sample, since the crucible was heated ( figures 5 to 8 ) before pouring the melt and then the furnace was switched off after stirring operation but the crucible was left in the furnace to cool down till it reached below eutectoid temperature. After that the crucible was taken out and cooled in ambient conditions. The conversion from perlite to ferrite matrix can also be caused due to coarseness of structure, the finer the structure lesser is the diffusion distance which favors the ferritic matrix. Diffusion is also affected by the cooling rate; lower cooling rate gives large time for diffusion. Also since the nodule 
count is increased due to the increase in the amount of nuclei available. This decreases the diffusion distance of carbon atoms, hence more carbon is diffused out of the matrix, increasing the area fraction of graphite precipitated. It seems larger amount of the carbon in stirred area has precipitated out of the matrix, due to the transformation of austenite phase to the ferrite phase, which can lower the carbide and martensite precipitation. Increase in volume fraction might also be a result of more ferrite in the matrix as can be observed in samples ROS 4 and 5.

During stirring the $\mathrm{Mg}$ present in the melt reacted with the environment and evaporated, this is also verified due to the fact during stirring lots of sparks were observed coming from the melt. Also the oxygen content of the melt increases relative to the $\mathrm{Mg}$. content. That could be the reason for the formation of graphite shapes other than nodular. So we observe the decrease in nodularity in general and where the stirring was continued for longer time period the graphite has precipitated in other forms.

\section{Conclusion}

By stirring we can increase the number of nuclei which increases the nucleation sites for graphite precipitation thereby increase the nodule count and precipitation of the graphite phase and transform the structure of nodular cast iron, hence affecting the mechanical properties of the alloy. More experiments are needed to be conducted to fully understand the mechanism and effects of stirring on the melt. Also there is need to find the optimum conditions.

\section{References}

[1] I. T. Im, W. S. Kim, K.S. Lee, A unified analysis of filling and solidification in casting with natural convection, Int. J. Heat Mass Tran. 44 (2001) 1507-1515.

[2] N. P. Yadav, Deepti Verma, Prediction of Solidification Behavior of Al Alloy in a Cube Mold Cavity, International Journal of Materials and Metallurgical Engineering 9 (No:12) (2015) 14181426

[3] Hasse Fredriksson, Ulla Åkerlind, "Materials Processing During Casting”. ISBN: 978-0-47001514-8

[4] C. R. Swaminathan, V. R. Voller, A time-implicit filling algorithm, Appl. Math. Model. 18 (1994) 101-108.

[5] S.N. Lekakh, J. Qing, V.L. Richards, K.D. Peaslee, Graphite Nodule Size Distribution in Ductile Iron, AFS Proceedings 2013 - American Foundry Society, Schaumburg, IL. Panel 13-1321.

[6] ASM Specialty Handbook Cast Irons: Editor: J.R. Davis, 1996, ISBN: 978-0-87170-564-8

[7] Spheroidal graphite cast irons - Classification, ISO 1083:2004

[8] Janina M. Radzikowska. Metallography and Microstructures, ASM Handbook Volume 9, 2004, pp. $565-587$

[9] F. Neumann, Giesserei 83 (1996) 11-15

[10] J. Müller, W. Siefer, Giessereiforschung 45 (1993) 92-98

[11] Qin Ziqiang, Yu Zongsen, Mechanism of inoculation of cast iron, Acta Metall Sin, 1 (1988) 67-71.

[12] Hasse Fredriksson, Ulla Akerlind, Solidification and Crystallization Processing in Metals and Alloys, 2012, ISBN: 978-1-119-99305-6. Page 175-182

[13] G.M. Goodrich, Cast Iron Microstructure Anomalies and Their Causes, AFS Trans. 105 (1997) 669-684

[14] H. Muhammad, F. Khan, A. Jutner, H. Fredriksson, Structure investigation of downhill and uphill casted inserts. Accepted for publication in SKB journal, 2017.

[15] H.J.T. Ellingham, Transactions and Communication, J. Soc. Chem. Ind. 63 (5) (1944) 125-160, doi:10.1002/jctb.5000630501. 